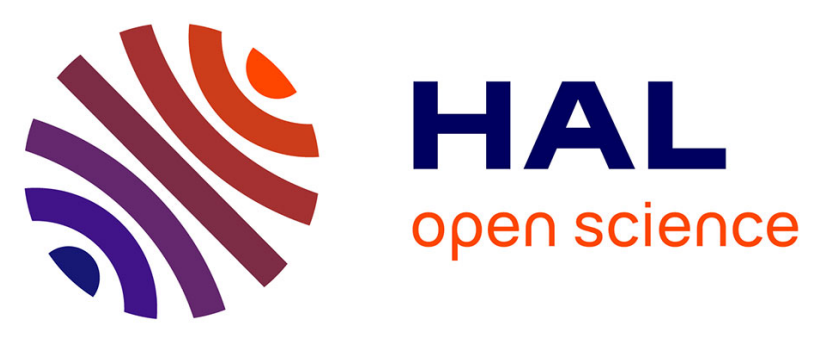

\title{
Defect Tolerance of Intersubband Transitions in Nonpolar GaN/(Al,Ga)N Heterostructures: A Path toward Low-Cost and Scalable Mid- to Far-Infrared Optoelectronics
}

Morteza Monavarian, Jiaming Xu, Michel Khoury, Feng Wu, Philippe de

Mierry, Philippe Vennegues, Mikhail A Belkin, James S Speck

\section{To cite this version:}

Morteza Monavarian, Jiaming Xu, Michel Khoury, Feng Wu, Philippe de Mierry, et al.. Defect Tolerance of Intersubband Transitions in Nonpolar GaN/(Al,Ga)N Heterostructures: A Path toward Low-Cost and Scalable Mid- to Far-Infrared Optoelectronics. Physical Review Applied, 2021, 16, 10.1103/physrevapplied.16.054040 . hal-03448129

HAL Id: hal-03448129

https://hal.science/hal-03448129

Submitted on 25 Nov 2021

HAL is a multi-disciplinary open access archive for the deposit and dissemination of scientific research documents, whether they are published or not. The documents may come from teaching and research institutions in France or abroad, or from public or private research centers.
L'archive ouverte pluridisciplinaire HAL, est destinée au dépôt et à la diffusion de documents scientifiques de niveau recherche, publiés ou non, émanant des établissements d'enseignement et de recherche français ou étrangers, des laboratoires publics ou privés. 


\title{
Defect Tolerance of Intersubband Transitions in Nonpolar GaN/(Al, Ga)N Heterostructures: A Path toward Low-Cost and Scalable Mid- to Far-Infrared Optoelectronics
}

\author{
Morteza Monavarian, $,{ }^{1,2,},{ }^{*} \S$ Jiaming Xu,${ }^{3, \S}$ Michel Khoury, ${ }^{2}$ Feng Wu,,${ }^{1,2}$ Philippe De Mierry, ${ }^{4}$ \\ Philippe Vennegues $\odot,{ }^{4}$ Mikhail A. Belkin, ${ }^{3,5, \dagger}$ and James S. Speck ${ }^{1,2, \$}$ \\ ${ }^{1}$ Materials Department, University of California, Santa Barbara, California 93106, USA \\ ${ }^{2}$ Solid-State Lighting Energy Electronic Center (SSLEEC), University of California, Santa Barbara, California \\ 93106, USA \\ ${ }^{3}$ Electrical and Computer Engineering Department, University of Texas, Austin, Texas 78758, USA \\ ${ }^{4}$ CNRS, Université Côte d'Azur, CRHEA, Rue Bernard Gregory, 06560 Valbonne, France \\ ${ }^{5}$ Walter Schottky Institute, Technical University of Munich, Am Coulombwall 4, 85748 Garching, Germany
}

(Received 28 June 2021; revised 6 October 2021; accepted 27 October 2021; published 22 November 2021)

We report on the impact of structural defects on mid-infrared intersubband (ISB) properties of $\mathrm{GaN} /(\mathrm{Al}, \mathrm{Ga}) \mathrm{N}$ heterostructures grown by ammonia molecular beam epitaxy $\left(\mathrm{NH}_{3} \mathrm{MBE}\right)$. Twenty-period $\mathrm{GaN} /(\mathrm{Al}, \mathrm{Ga}) \mathrm{N}$ multi-quantum-well (MQW) heterostructures are grown on co-loaded $a$-plane freestanding GaN substrates and heteroepitaxial $a$-plane $\mathrm{GaN}$ on $r$-plane sapphire templates $(a$ - $\mathrm{GaN} / r$-sap) for three different quantum-well $(\mathrm{QW})$ widths $(3.0,3.3$, and $3.7 \mathrm{~nm})$. Co-loaded structures grown on freestanding $a$-plane with no basal-plane stacking faults (BSFs), prismatic stacking faults (PSFs), and partial dislocations (PDs), with low threading dislocation (TD) densities of about $10^{5} \mathrm{~cm}^{-2}$ are compared with those grown on $a$-GaN templates on (1012) $r$-sapphire with BSF, PSF, PD, and TD densities of about $4 \times 10^{5}$ to $10^{6} \mathrm{~cm}^{-2}, 5 \times 10^{3}$ to $2 \times 10^{4} \mathrm{~cm}^{-2}$, about $9 \times 10^{10}$ to $2 \times 10^{11} \mathrm{~cm}^{-2}$, and about $10^{10} \mathrm{~cm}^{-2}$, respectively. Fourier-transform infrared absorption spectroscopy indicates ISB transition energies in the range of about 250-300 meV (wavelength range 4.1-4.8 $\mu \mathrm{m}$ ) for MQWs with different QW widths. The ISB absorption spectra indicate about 5\% smaller transition energies and only about 10\%-20\% larger spectral linewidths for structures grown on $a-\mathrm{GaN} / r$-sapphire templates compared with those on freestanding $\mathrm{GaN}$ substrates. The strong defect tolerance in the nonpolar $a$-plane ISB structures could be due to the nature of defects and their energy levels with respect to the conduction-band minima, which do not affect the ISB properties. Our results pave the way toward the production of low-cost scalable nonpolar III-nitride MQW heterostructures for a variety of passive and active optical materials and devices based on intersubband transitions.

DOI: 10.1103/PhysRevApplied.16.054040

\section{INTRODUCTION}

Since the discovery and implementation of quantumcascade lasers (QCLs) in the mid-1990s [1], intersubband (ISB) transitions have been widely considered for mid-infrared (mid-IR, $\lambda \approx 3-30 \mu \mathrm{m})$ and terahertz (THz, $\lambda \approx 30-300 \mu \mathrm{m}$ ) devices for various applications, including biological imaging, telecommunications, pharmaceutical quality control, and security screening [2,3]. Unlike conventional semiconductor lasers [4] that rely on the recombination of electron-hole pairs via band-to-band

\footnotetext{
*mmonavarian@ucsb.edu

†mikhail.belkin@wsi.tum.de

¥speck@ucsb.edu

$\S$ These authors contributed equally to this work.
}

transitions, QCLs are unipolar devices with lasing obtained via ISB transitions in a stack of multi-quantum-well (MQW) heterostructures [5]. THz QCLs based on arsenide and phosphide systems face issues of small longitudinal optical (LO) phonon energies [6], especially for higher frequencies $(5-10 \mathrm{THz})$. Group-III nitrides, on the other hand, have the advantage of large LO phonon energy ( $\sim 92 \mathrm{meV}$ ) [7], which make them potentially more favorable for room-temperature QCLs across the entire $\mathrm{THz}$ spectral range $[8,9]$. In addition, due to the large band offsets $[7,10,11]$, III nitrides may provide access to ranges of wavelengths inaccessible to devices and optical materials based on intersubband transitions in the arsenide- and phosphide-based material systems [12].

Nonlinear optical and electro-optic elements based on ISB transitions [13-20] can also benefit from 
the wider accessible range of wavelengths provided by the high-conduction-band-offset III-nitride systems. To enhance optical nonlinearities, using wave-guiding schemes [21,22], metallic nanoantenna arrays [23], and nonlinear metasurface-based flat optical components [15-17,24,25] has received increasing attention. In particular, the introduction of subwavelength thickness nonlinear metasurfaces provides a path toward efficient frequency mixing without the need for phase-matching limitations of bulk nonlinear crystals [26] and with controlled output-beam phase front [17,27]. Application of QCLs and nonlinear frequency conversion, such as secondharmonic generation (SHG) $[18,19]$, sum-frequency generation (SFG) [20], and difference-frequency generation (DFG) [20], with enhanced nonlinearities in III-nitridebased systems can provide light sources over wide ranges of wavelengths from near- to far-IR.

Conventional III-nitride materials on polar (0001) orientation ( $c$ plane) face various limitations due to the strong polarization-induced internal electric field [28]. The electric field across the active QWs in ISB structures can markedly reduce electron-wave-function overlap $[29,30]$, ISB-transition oscillator strength, and thus transition efficiency $[12,31,32]$. In addition, the polarizationrelated electric field normally distorts the band profiles to shape triangular QWs, resulting in the escape of electrons from the active region [32]. Nonpolar crystal orientation of III nitrides [Fig. 1(a)] with suppressed polarizationrelated electric field is an alternative to eliminate the abovementioned issues with conventional polar orientation $[12,28]$.

There are different reports on nonpolar III nitrides (either $\{10 \overline{1} 0\}$ family of $m$ planes or nonpolar $\{11 \overline{2} 0\}$ family of $a$ planes) for ISB applications [31,33-37]. We recently reported a narrow ISB absorption linewidth of about $38 \mathrm{meV}$ at $4.5-5.5 \mu \mathrm{m}$ on nonpolar (1010) ( $m$-plane) freestanding substrates and systematically compared the two nonpolar planes for ISB applications [12,38]. Most of the reported nonpolar III-nitride ISB properties are on freestanding substrates, which are extremely expensive and small in size $[28,39,40]$. The lack of high-quality inexpensive nonpolar substrates has been a bottleneck in regular band-to-band-based applications, such as light-emitting diodes (LEDs) and lasers [28]. However, thus far, there is no clear indication as to whether ISB properties are similarly affected by the presence of extended defects compared with the band-to-band transitions, especially since most of the extended defects cause nonradiative centers as states within the band gap and, thus may not affect the ISB structures, which are unipolar in nature.

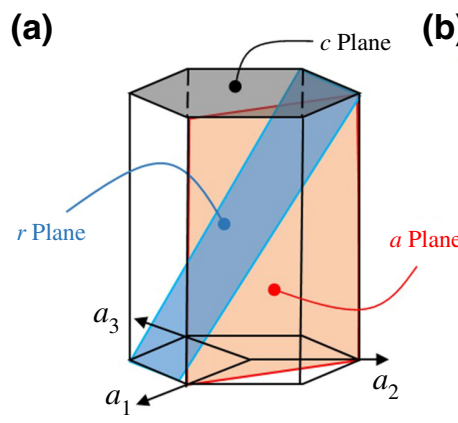

(c)

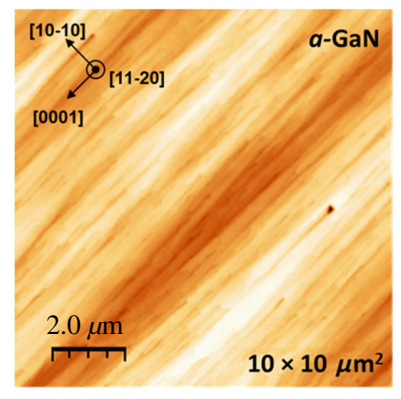

(b)

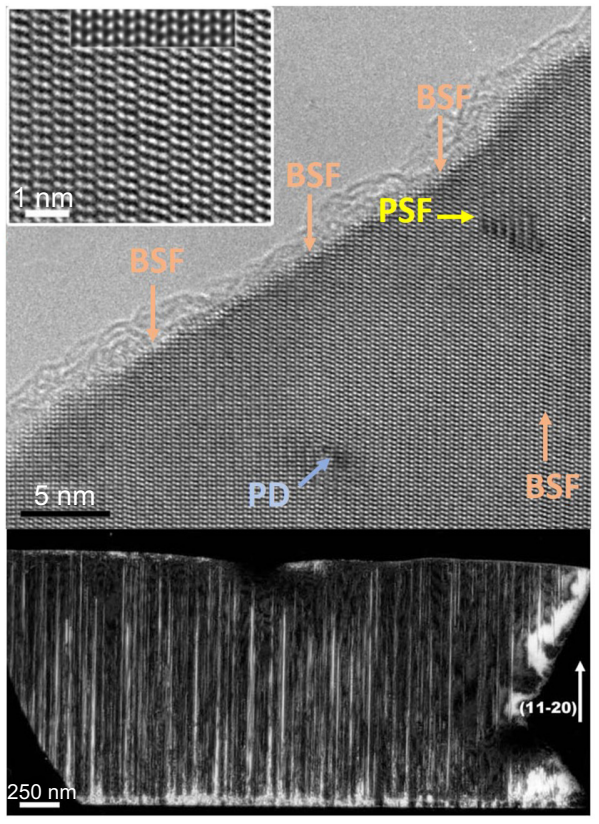

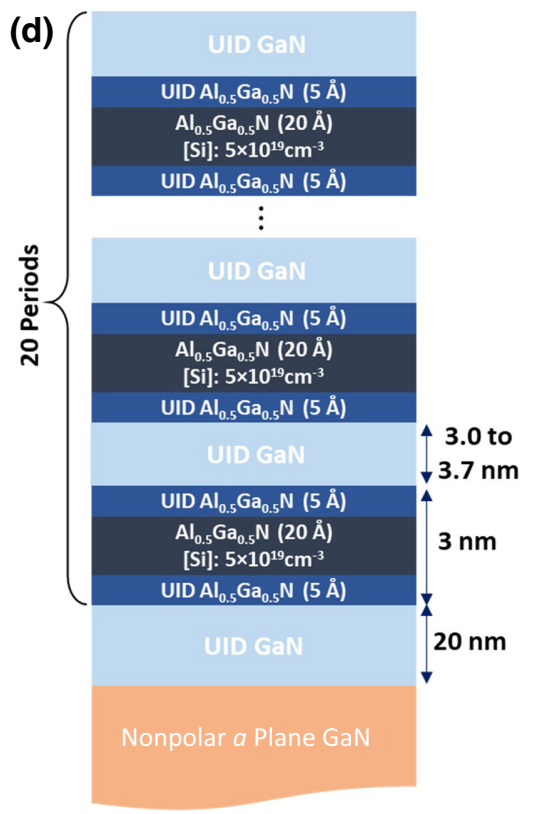

FIG. 1. (a) Schematic representation of selected crystal planes in wurtzite crystal structure of III nitrides, indicating polar $c$ plane, semipolar $\{10 \overline{1} 2\} r$ plane, and nonpolar $\{11 \overline{2} 0\}$ a plane. (b) Plan-view (top) high-resolution transmission electron microscopy (TEM) image of the $a$-GaN/r-sap template along the [1120]-zone axis, showing that dominant defects are basal-plane stacking faults (BSFs), which are indicated by orange arrows. BSFs may either be terminated by partial dislocation (PDs) (pale blue arrows), or two neighboring BSFs may be connected by a (1120) PSF (yellow arrow). Bottom of (b) shows a cross-section image of PDs that are vertically threading from the interface to the surface. (c) Representative surface of $a$-plane orientation of GaN, indicating elongated features toward $c$ direction. (d) Schematic structure of $a$-plane GaN/(Al, Ga)N ISB structures used in this study. Notably, monolayer thickness for $\mathrm{GaN}$ is about $0.25 \mathrm{~nm}$. 
Here, we systematically compare the ISB transition properties of $\mathrm{GaN} /(\mathrm{Al}, \mathrm{Ga}) \mathrm{N}$ MQW heterostructures on nonpolar $a$-plane orientation on substrates with low and high defect densities.

\section{EXPERIMENTAL DETAILS}

The structures are all grown by Gen 930 ammonia molecular beam epitaxy $\left(\mathrm{NH}_{3} \mathrm{MBE}\right)$, where $\mathrm{NH}_{3}$ decomposition at elevated temperatures provides elemental $\mathrm{N}$ for growth of $\mathrm{GaN}$ and its alloys. In the $\mathrm{NH}_{3} \mathrm{MBE}$ system, $\mathrm{Ga}$ and $\mathrm{Al}$ effusion cells are used to provide metallic elements and a $\mathrm{Si}$ effusion cell is used for $\mathrm{Si} n$-type dopant. Two sets of samples are considered. The first set of samples use freestanding (FS) $a$-GaN from the Mitsubishi Chemical Corporation (MCC) with no BSFs, PSFs, and PDs, and a TD density of about $10^{5} \mathrm{~cm}^{-2}$. The second set of samples utilize heteroepitaxial $a$-plane $\mathrm{GaN}$ templates grown on $r$-plane sapphire ( $a$-GaN/r-sap) (the most commonly used foreign substrate for the growth of nonpolar $\mathrm{GaN}$ ). The heteroepitaxy of $a$-GaN/r-sap templates is carried out by metalorganic chemical vapor deposition (MOCVD) in a close-coupled showerhead reactor using trimethylgallium and $\mathrm{NH}_{3}$ as precursors and hydrogen $\left(\mathrm{H}_{2}\right)$ as the main carrier gas. The $0.85-\mu \mathrm{m}$-thick $a$-plane $\mathrm{GaN}$ templates are grown on $r$-plane sapphire. The epitaxial process begins with sapphire nitridation at $1120^{\circ} \mathrm{C}$ and low-temperature $\mathrm{GaN}$ buffer deposition at $690^{\circ} \mathrm{C}$ at 100 Torr, followed by buffer annealing and three-dimensional (3D) crystal formation at $1200{ }^{\circ} \mathrm{C}$ and 700 Torr, and crystal expansion at $1090^{\circ} \mathrm{C}$ and 300 Torr, before the final coalescence step at $1200^{\circ} \mathrm{C}$ and 10 Torr.

The microstructure of nonpolar heteroepitaxial films is very different from those deposited along the polar $c$ direction, which is dominated by the presence of vertically threading perfect dislocations mainly of $a$ type (edge) and $a+c$ type (mixed). Even though the densities and nature of extended defects in heteroepitaxial nonpolar GaN may be a bit different, depending on the substrates, similar general trends are observed for nonpolar $a-\mathrm{GaN} / r$-sap layers. Figure 1(b) shows a high-resolution cross-section TEM image of the $a$-GaN grown on $r$-sapphire by MOCVD, indicating the different types of defects. The cross-section images clearly show extensive TDs, BSFs terminated by PDs, and PSFs. The densities of the different defects in these samples are about $4 \times 10^{5}-1 \times 10^{6} \mathrm{~cm}^{-1}$ for BSFs, about $9 \times 10^{10}$ to $2 \times 10^{11} \mathrm{~cm}^{-2}$ for PDs, and about $5 \times 10^{3}$ to $2 \times 10^{4} \mathrm{~cm}^{-1}$ for PSFs. The surface morphology of $a$-GaN/r-sap is also shown in Fig. 1(c), indicating typical elongated cometlike features along the $c$ directions. The

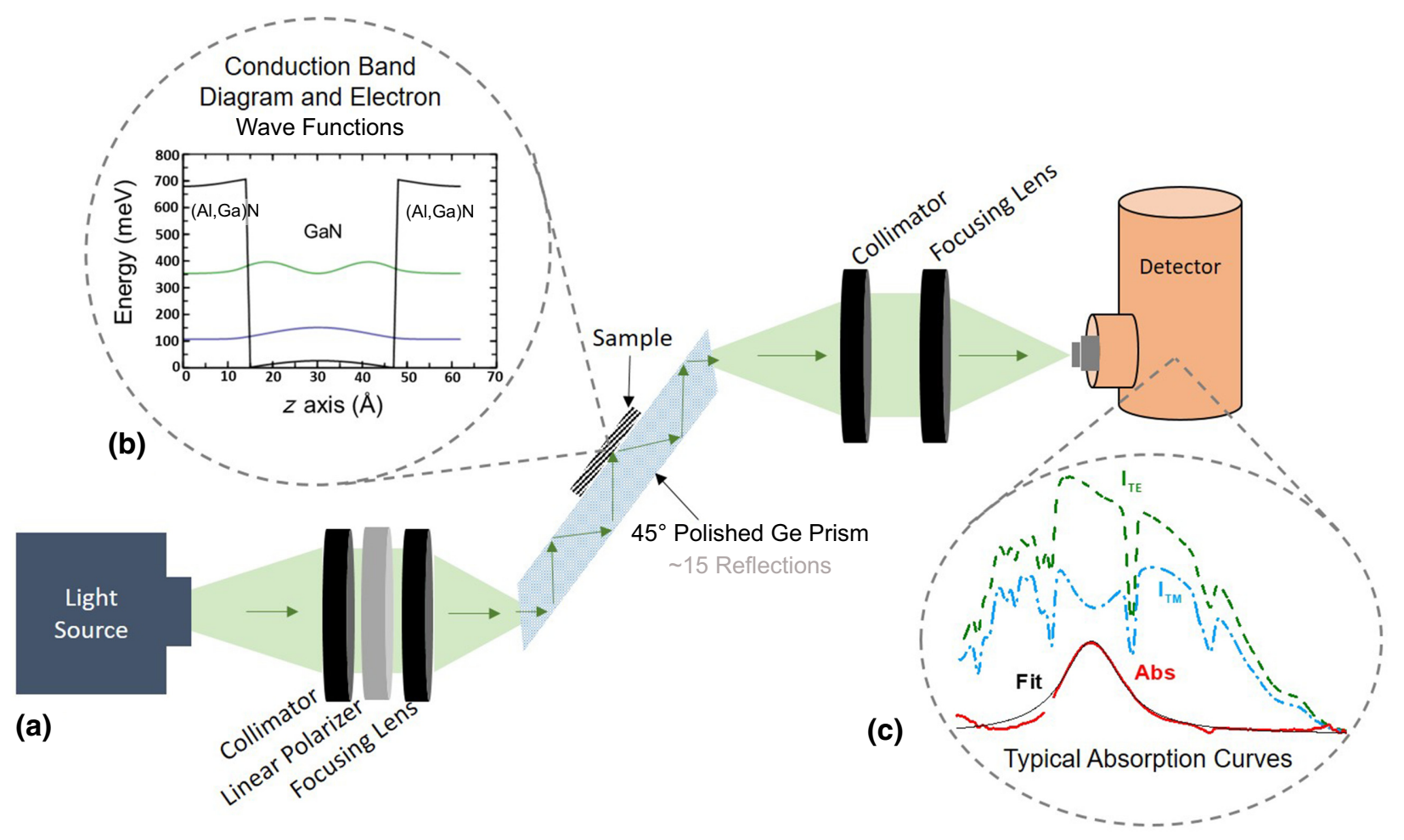

FIG. 2. (a) Schematic of the setup to measure ISB absorption in our samples. Samples are placed epi-side-down onto an IRtransparent Ge prism. (b) Calculated conduction-band diagram and square of electron wave functions of the first-two quantum states for the MQW sample with a 3.3-nm QW width. Transmitted signal for two different polarizations of incident light (TE and TM) are captured. (c) Sample plots of absorption spectra for TE- and TM-polarized incident light and the corresponding ISB absorption. 
diagonal lines across the atomic force microscopy (AFM) images are along the $+c$ [0001] direction and at $90^{\circ}$ to the $m$ direction, [1010].

The ISB structures are then grown on co-loaded FS $a$-GaN and $a$-GaN/r-sap substrates. Figure 1(d) schematically shows the detailed ISB structures grown for this investigation. The structures consist of 20 periods of nominal $\mathrm{GaN} / \mathrm{Al}_{0.4} \mathrm{Ga}_{0.6} \mathrm{~N}$ MQW heterostructures on a 20-nm unintentionally doped (UID) GaN buffer layer. The number of QWs in the MQW stack is chosen so that the stack thickness is similar to the penetration depth in our attenuated total-internal-reflection absorption measurements that are described further below. Three different sets of structures are grown, for which the thickness of GaN QWs are changed (3.0, 3.3, and $3.7 \mathrm{~nm})$. For all the structures, the thicknesses of the $\mathrm{Al}_{0.4} \mathrm{Ga}_{0.6} \mathrm{~N}$ quantum barriers $(\mathrm{QBs})$ are kept constant at $3.0 \mathrm{~nm}$. Of the 3-nm QBs, the bottom and top $0.5 \mathrm{~nm}$ of the QBs are kept UID, while the innermost $2.0 \mathrm{~nm}$ of the $\left(\mathrm{Al}_{0.4} \mathrm{Ga}_{0.6} \mathrm{~N}\right.$ QBs are doped with $\mathrm{Si}$ (up to $\sim 5 \times 10^{19} \mathrm{~cm}^{-3}$ ). The doping level is chosen to achieve a high doping concentration to produce large intersubband absorption, while keeping the Fermi energy $\left(E_{F}\right)$ for a two-dimensional (2D) electron gas in a $\mathrm{QW}$ at least $100 \mathrm{meV}$ below the ISB transition energy to minimize electron population of the first excited level in the MQW system. Details of the calculations are provided in the Supplemental Material [41].

The structures are then characterized using highresolution x-ray diffraction (XRD), TEM or scanning TEM (STEM), AFM, and atom-probe tomography (APT). To evaluate the interface quality, the QW thicknesses, and characterization of the extended defects, high-angle annular dark-field (HAADF) STEM is used. The TEM or STEM samples are prepared using a focused ion beam (FIB) with an FEI Helios DualBeam Nanolab 600 instrument. A ThermoFisher Talos G2 200X TEM-STEMwChemiSTEM energy-dispersive spectroscopy (EDS) system operating at $200 \mathrm{kV}$ is used to perform microstructure and chemical-element mapping. APT is also used to investigate the compositions and alloy distribution in the $\mathrm{GaN} /(\mathrm{Al}, \mathrm{Ga}) \mathrm{N}$ heterostructures in $3 \mathrm{D}$ at the nanometer scale $[42,43]$. The needle-shaped specialized APT samples are prepared using a FEI Helios 600 dual-beam FIB instrument [44], and the analysis is performed in laserpulse mode using a Cameca 3000X HR local-electrode atom probe (LEAP). A laser wavelength of $532 \mathrm{~nm}, 13$-ps pulse duration, $200-\mathrm{kHz}$ repetition rate, $10-\mu \mathrm{m}$ spot size, $0.5-\mathrm{nJ}$ pulse energy, and detection rate of 0.02 atoms per pulse are used for the APT measurement analysis, while the sample base temperature is $30 \mathrm{~K}$. The commercial IVAS $^{\mathrm{TM}}$ software is used to obtain the 3D reconstruction, where a geometrical algorithm is used based on the initial radius and the shank angles of the prepared tips, according to the images obtained from scanning electron microscopy data [44].

Finally, the ISB absorption properties of the samples are measured using a Fourier-transform IR (FTIR) spectroscopy transmission setup equipped with a mercury cadmium telluride (MCT) infrared photodetector. The measurement setup schematic is shown in Fig. 2. Due to strong substrate absorption, we use evanescent excitation of the $\mathrm{GaN} /(\mathrm{Al}, \mathrm{Ga}) \mathrm{N}$ QWs brought in optical contact with $350-\mu \mathrm{m}$-thick $45^{\circ}$-polished Ge prisms (attenuated totalinternal-reflection absorption measurements). Given that the refractive indices of $\mathrm{GaN}$, nominal $\mathrm{Al}_{0.4} \mathrm{Ga}_{0.6} \mathrm{~N}$, and Ge are known to be approximately $2.25,2.1$, and 4.0, respectively, and the light incident angle of $45^{\circ}$ on the Gesample interface, we find that the intensity of the evanescent illumination extends approximately $0.2 \mu \mathrm{m}$ into the MQW structure for the measurement wavelength range of 4-5 $\mu \mathrm{m}$. Since the thicknesses of the MQW stack in our
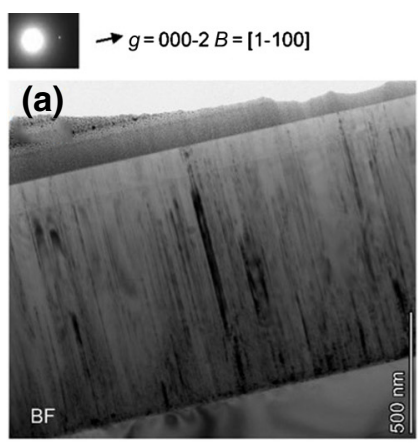

. $\downarrow g=-1-120 \quad B=[1-100]$

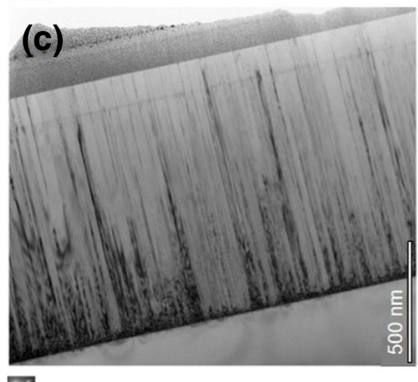

$g=01-10 B=[2-1-10]$

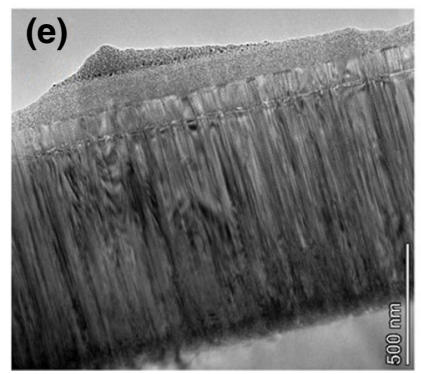

FIG. 3. Cross-section TEM images of $\mathrm{GaN} /(\mathrm{Al}, \mathrm{Ga}) \mathrm{N} \mathrm{MQW}$ structures on $a-\mathrm{GaN} / r$-sap with (a),(c),(e) two-beam BF diffraction contrast and (b),(d) weak-beam DF diffraction contrast along (a),(b) $g=000 \overline{2}$, (c),(d) $g=\overline{1} \overline{1} 20$, and (e) $g=01 \overline{1} 0$. Scale bars are the same for all the images. 
samples are in the range of 120-135 nm, a nearly uniform illumination of the MQWs is achieved. The ISB absorption spectra are obtained by normalizing TM-polarized transmission spectra with TE-polarized transmission spectra that provide a reference, since TE-polarized light does not interact with ISB transitions. A Lorentz function is used to fit the ISB absorption spectra to obtain the peak energy and spectral linewidth.

\section{RESULTS AND DISCUSSION}

Figure 3 shows the structural defects in nominal $\mathrm{GaN} / \mathrm{Al}_{0.4} \mathrm{Ga}_{0.6} \mathrm{~N}$ MQWs grown on $a-\mathrm{GaN} / r$-sap in the (a)

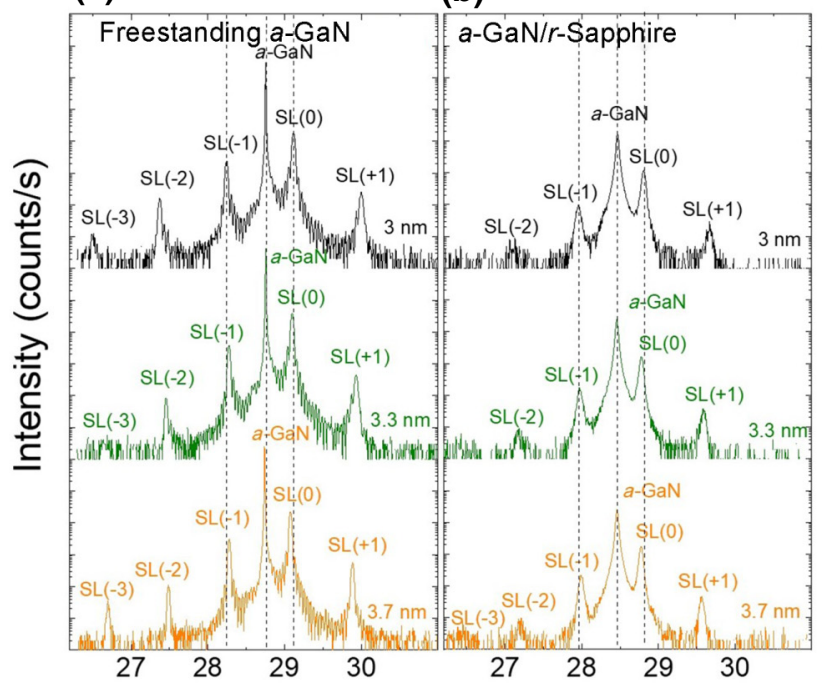

(c)

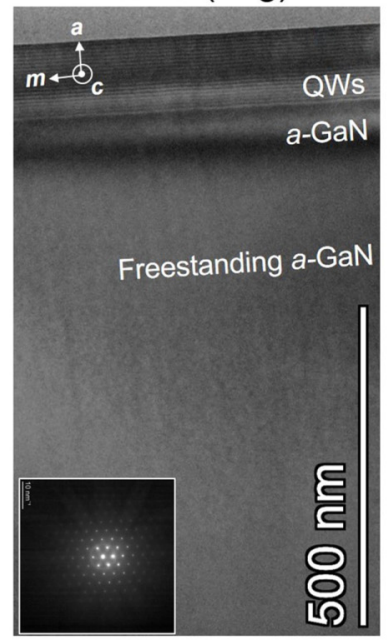

(d)

$\omega-2 \theta$ (deg)

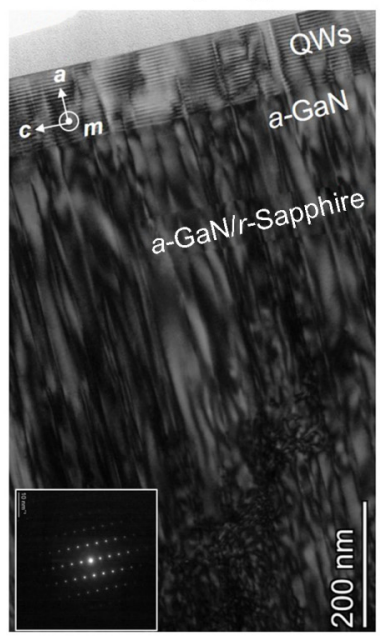

FIG. 4. High-resolution (a),(b) XRD $\omega-2 \theta$ scans and (c),(d) cross-section HAADF TEM images of structures on (a),(c) FS $a$-GaN and (b),(d) $a-\mathrm{GaN} / r$-sap. In the XRD measurements, the azimuthal angle is chosen in such a way that the (2204) $r$-sapphire peak (at $\sim 27.7^{\circ}$ ) is not captured. Also, note the difference in the axes of observation for the TEM images of (c),(d); the results are not dependent on this. cross section along the [1100] direction using two-beam bright-field (BF) diffraction contrast [Figs. 3(a), 3(c), and 3(e)] and weak-beam dark-field (DF) diffraction contrast [Figs. 3(b) and 3(d)]. The results indicate that all dislocations are located in the $c$ plane. The TEM images illustrate the highly defective microstructure of $a-\mathrm{GaN} / r$ sap, including the ISB GaN/ $\mathrm{Al}_{0.4} \mathrm{Ga}_{0.6} \mathrm{~N}$ QWs. While it is difficult to evaluate the accurate densities of different types of defects from these TEM images, we confirm that $a+c$ are in contrast with partial dislocations for $g=0002$, while $a+c, a$, and $2 / 3$ of all partial dislocations are in contrast for $g=11 \overline{2} 0$. The microstructure of the $a-\mathrm{GaN} / r$-sap structures is similar to that we reported previously [45].

Figure 4 compares the structural properties of the epilayers grown on FS $a$-GaN and on $a$-GaN/r-sap using high-resolution XRD $\omega-2 \theta$ scans and cross-section TEM. XRD data indicates sharp GaN peaks for the samples on FS $a$-GaN [Fig. 4(a)], while much weaker GaN peaks are observed for all the structures grown on $a-\mathrm{GaN} / r$ sap ( 2 to 3 orders of magnitude lower peak intensity) [Fig. 4(b)]. The MQW structures grown on FS $a$-GaN also show more pronounced satellite (SL) peaks (five intense SL peaks), as shown in Fig. 4(a), which are normally observed for short-period superlattices [46]. In contrast, the structures grown on $a$-GaN $/ r$-sap only show four less pronounced SL peaks, as shown in Fig. 4(b). These results

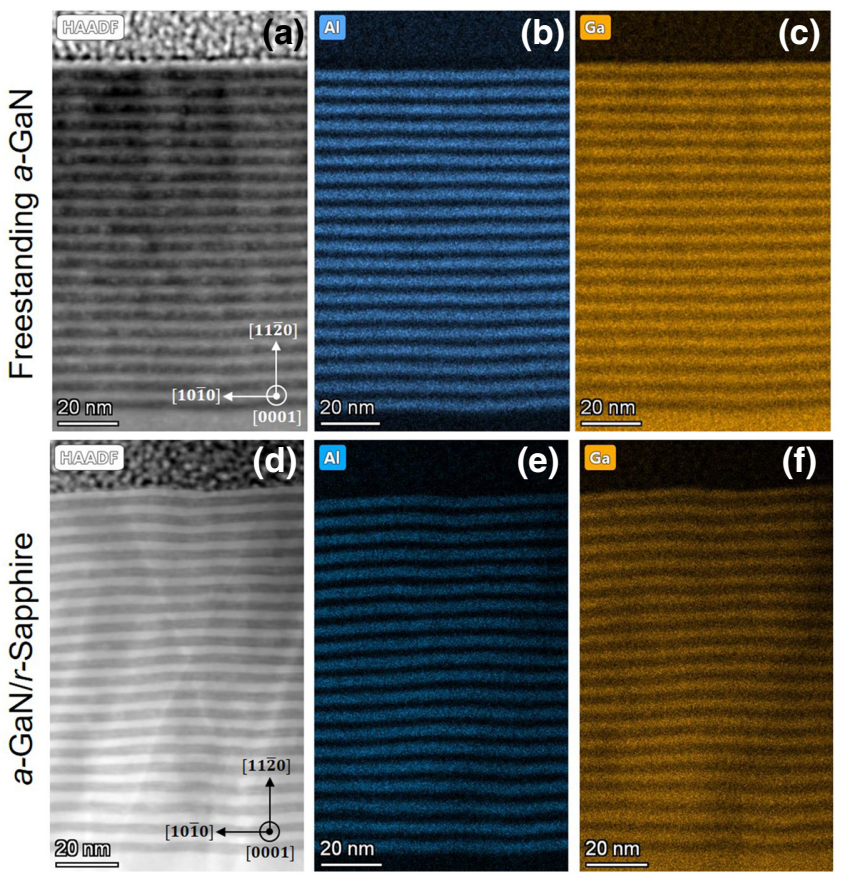

FIG. 5. HAADF (a),(d) cross-section STEM images across (0001) $c$ plane and corresponding (b),(e) Al and (c),(f) Ga elemental maps of $\mathrm{GaN} /(\mathrm{Al}, \mathrm{Ga}) \mathrm{N}$ MQW heterostructures with 3.0-nm QWs grown by $\mathrm{NH}_{3} \mathrm{MBE}$ on (a)-(c) $\mathrm{FS} a-\mathrm{GaN}$ and (d)-(f) $a-\mathrm{GaN} / r$-sap substrates. 
(a)

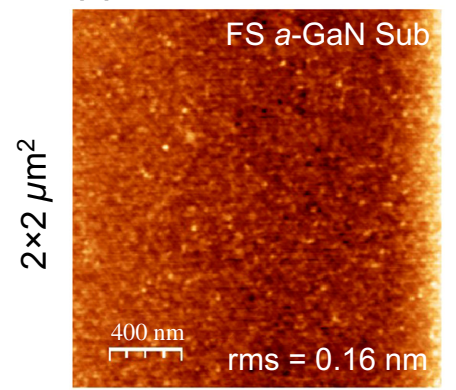

(b)

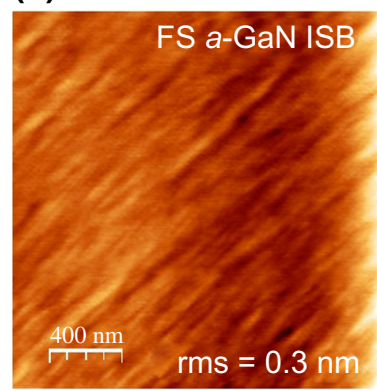

(c)

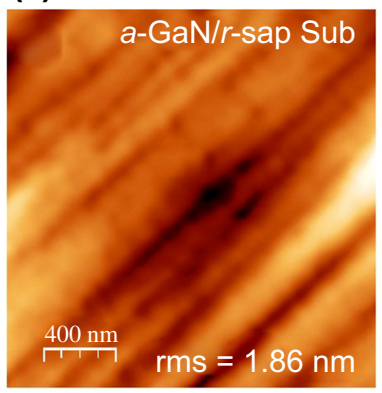

(d)

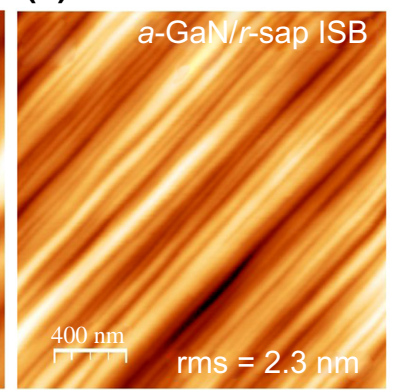

FIG. 6. $2 \times 2 \mu \mathrm{m}^{2}$ AFM images of (a) FS $a$-GaN substrate, (b) ISB GaN/(Al, Ga)N heterostructures grown on FS $a$-GaN substrate, (c) $a$-GaN/r-sap substrate, and (d) ISB GaN/(Al, Ga)N heterostructures grown on $a-\mathrm{GaN} / r$-sap substrate. Elongated features observed for structures on $a$-GaN/ $r$-sap (c),(d) correspond to the high density of stacking faults, while no elongated features are observed for the FS structures (a),(b) (no stacking faults).

are indicative of higher interface abruptness for the structures grown on FS $a$-GaN. The period of the SL peaks consistently reduces for thicker QW structures for both cases, consistent with larger periodicity of the superlattice structure. A comparison of cross-section HAADF TEM [Figs. 4(c) and 4(d)] images of selected samples from

\section{(a)}

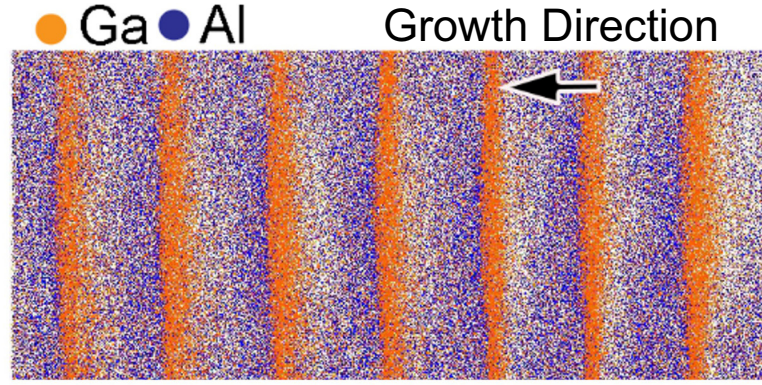

(b)

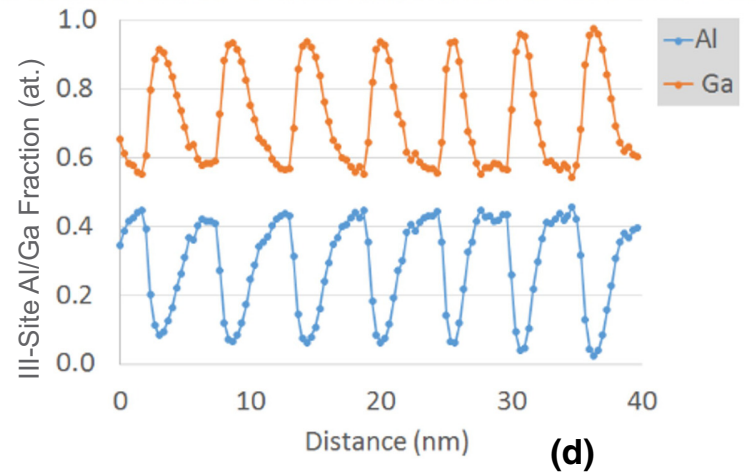

(c)

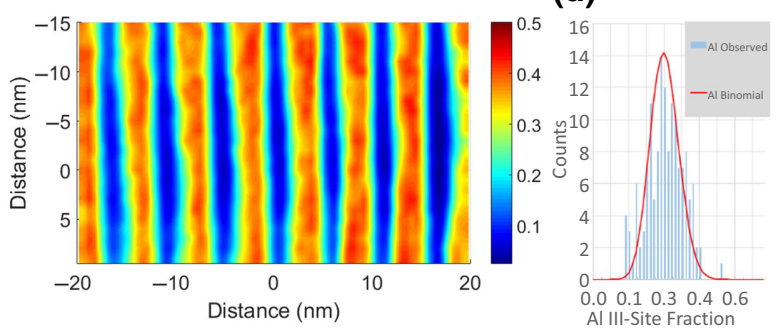

ISB on $a-G a N / r-S a p p h i r e$
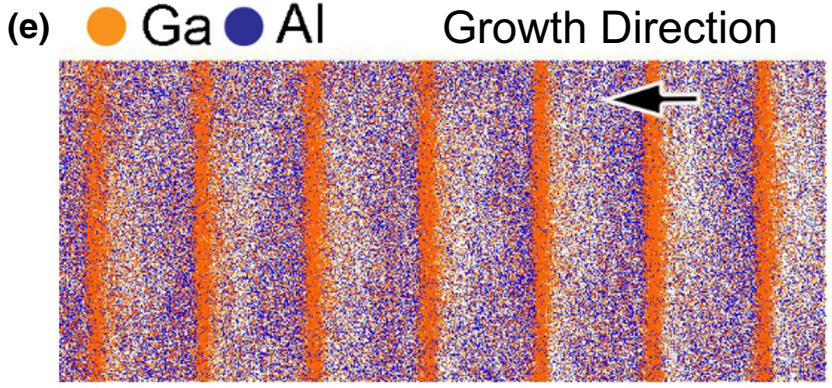

(f)

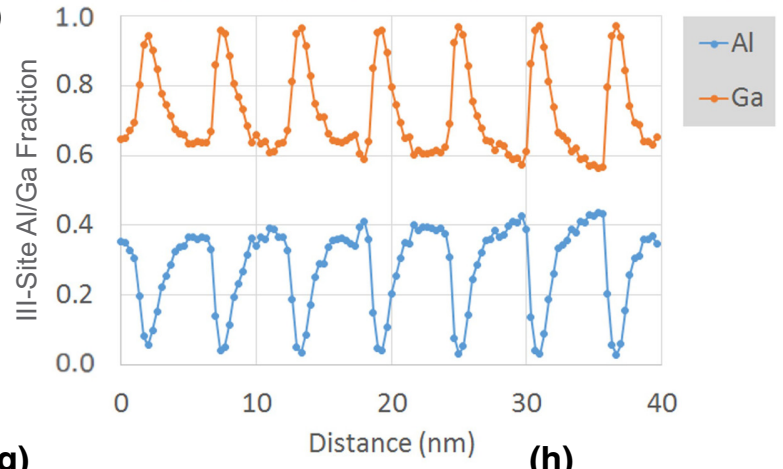

(g)

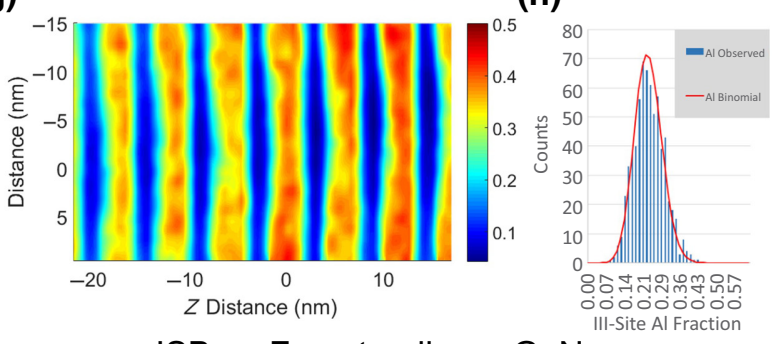

FIG. 7. (a),(e) APT data and analysis, (b),(f) atomic line profiles of $\mathrm{Ga}$ and $\mathrm{Al}$ concentrations, (c),(g) Al composition maps along the growth direction, and (d),(h) distribution of $\mathrm{Al}$ contents in the $(\mathrm{Al}, \mathrm{Ga}) \mathrm{N}$ QBs for ISB structures containing 20-period nonpolar $a$-plane $\mathrm{GaN} /(\mathrm{Al}, \mathrm{Ga}) \mathrm{N}$ MQW heterostructures on (a)-(d) $a$-GaN/r-sapphire and (e)-(h) freestanding $a$-GaN. The difference in distribution of $\mathrm{Al}$ contents in the barrier layers for the $a-\mathrm{GaN} / r$-sap sample compared with FS $a$-GaN may result in differences in the ISB absorption peak energy and linewidth. 
superlattices with 3.0-nm QW width indicate a large difference in the densities of defects extended to the MQW heterostructures between the two structures, as expected from their extremely different substrate qualities.

The ISB optical transition properties, such as linewidth, can be strongly dependent on the interface abruptness. Hence, the interface quality and surface morphology of the structures for 3.0-nm QW width grown on FS $a$-GaN and $a-\mathrm{GaN} / r$-sap are compared using cross-section STEM and EDS (Fig. 5) and AFM (Fig. 6). The structure grown on FS $a$-GaN shows a higher interface quality compared with the $a-\mathrm{GaN} / r$-sap sample. Also, the thicknesses of the QWs are slightly smaller for FS $a$-GaN $(\sim 2.9-3.0 \mathrm{~nm})$ compared with those of the $a-\mathrm{GaN} / r$-sap $(\sim 3.1$ to $3.2 \mathrm{~nm})$ samples, although this could be a projection effect, as the corrugated interface observed in the cross-section plane in Fig. 5 ( $c$ plane) may also exist in the plane perpendicular to the cross-section plane at which the STEM image is taken. The AFM results (Fig. 6) also indicate larger rootmean-square (rms) roughness values for the $a$ - GaN $/ r$-sap substrates $\left(1.86 \mathrm{~nm}\right.$ for $2 \times 2 \mu \mathrm{m}^{2}$ scans) compared with the FS $a-\mathrm{GaN}$ substrate $(0.16 \mathrm{~nm})$ and the corresponding ISB structure on $a-\mathrm{GaN} / r$-sap $(2.3 \mathrm{~nm})$ compared with the one grown on FS $a$-GaN $(0.3 \mathrm{~nm})$. A more detailed analysis of AFM data can be found in the Supplemental Material [41].

Figure 7 compares APT data and analysis of MQW samples with 3.0-nm QWs grown on $a$-GaN/r-sap and
FS $a$-GaN substrates. According to APT data, along the growth direction, the top interfaces between $\mathrm{GaN}$ and $(\mathrm{Al}, \mathrm{Ga}) \mathrm{N}(\mathrm{QW} / \mathrm{QB}$ interfaces) are slightly more abrupt than the bottom interfaces (Fig. 7), similar to our previous observations on other $\mathrm{GaN} /(\mathrm{Al}, \mathrm{Ga}) \mathrm{N}$ MQW structures [12]. As shown in the variations of $\mathrm{Ga} / \mathrm{Al}+\mathrm{Ga}$ and $\mathrm{Al} / \mathrm{Al}+\mathrm{Ga}$ profiles along the growth directions [Figs. 7(b) and 7(f)], the $\mathrm{Al}$ fraction in the (Al, Ga) N layers varies in the range of $38-45 \%$ with about $8 \%$ deviation for the structure grown on $a-\mathrm{GaN} / r$-sap and in the range of $38-40 \%$ with about $4 \%$ deviation for the structure grown on FS $a$-GaN. A normal Gaussian distribution of $\mathrm{Al}$ atoms in the (Al, Ga)N QBs for the structure grown on FS $a$-GaN [Fig. 7(h)] indicates a natural random alloy disorder without significant clustering, with an average composition of about $30 \%$. For the structure grown on $a-\mathrm{Ga} / r$-sap, however, the distribution of $\mathrm{Al}$ atoms is not as symmetric and does not fully obey a Gaussian model [Fig. 7(d)], which indicates alloy clustering, to some degree, while maintaining a similar average composition to that of the structure on FS $a-\mathrm{GaN}$.

The structures are then tested for optical absorption using the FTIR spectroscopy transmission setup described in Fig. 2. First, the transmitted intensity of the incident wide-band light source upon evanescently coupling to the MQW ISB structures is detected as a function of wavelength for TE- and TM-polarized incident light. The ISB absorption spectra are then computed by (a)
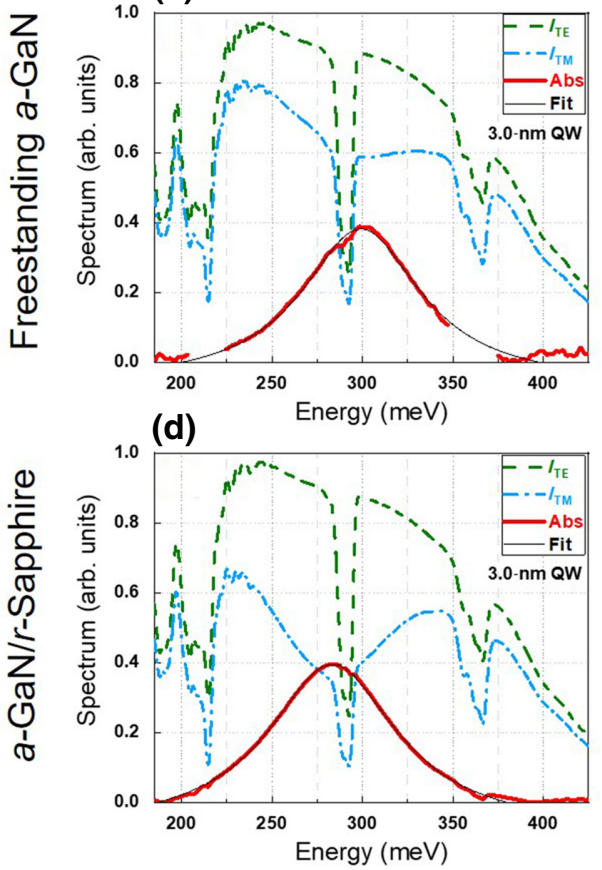

(b)

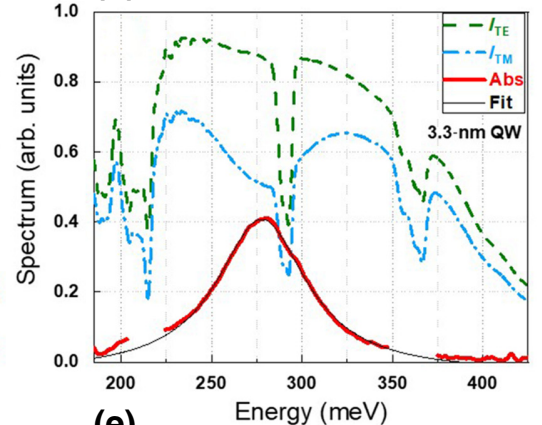

(e)

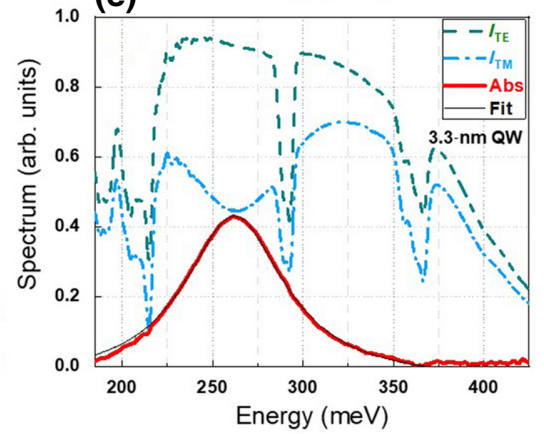

(c)
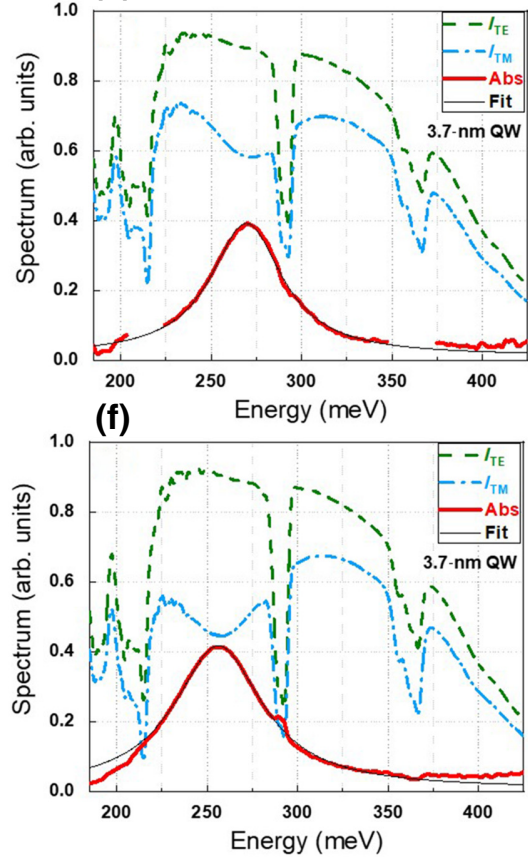

FIG. 8. Transmission spectra for TE-polarized (green dashed line) and TM-polarized (blue dashed-dotted line) incident light and the obtained ISB absorption spectra (thick red solid line) and Lorentz-function fitting curves (thin solid black line) for the MQW structures on (a)-(c) FS $a-\mathrm{GaN}$ and (d)-(f) $a-\mathrm{GaN} / r$-sap with QW widths of (a),(d) $3.0 \mathrm{~nm}$, (b),(e) $3.3 \mathrm{~nm}$, and (c),(f) $3.7 \mathrm{~nm}$. 
TABLE I. Experimentally measured and calculated ISB transition energies $\left(E_{12}\right.$ in meV) and absorption linewidths for the $\mathrm{GaN} /(\mathrm{Al}, \mathrm{Ga}) \mathrm{N}$ MQW structures grown on FS $a$-GaN and on heteroepitaxial $a$-GaN/ $r$-sap.

\begin{tabular}{|c|c|c|c|c|c|}
\hline \multirow[b]{2}{*}{ QW thickness (nm) } & \multicolumn{2}{|c|}{ Samples grown on FS $a-\mathrm{GaN}$} & \multicolumn{2}{|c|}{ Samples grown on $a-\mathrm{GaN} / r$-sap } & \multirow{2}{*}{$\begin{array}{l}\text { Theoretical energy } \\
\text { (meV) with many body }\end{array}$} \\
\hline & Energy $(\mathrm{meV})$ & Linewidth (meV) & Energy $(\mathrm{meV})$ & Linewidth (meV) & \\
\hline 3.0 & 299.1 & 80.7 & 283.5 & 87.4 & 299.25 \\
\hline 3.3 & 277.8 & 60.7 & 262.1 & 67.7 & 267.51 \\
\hline 3.7 & 269.6 & 48.4 & 256.2 & 59.6 & 233.13 \\
\hline
\end{tabular}

plotting $\ln \left(I_{\mathrm{TE}} / I_{\mathrm{TM}}\right)$, where $I_{\mathrm{TE}}$ and $I_{\mathrm{TM}}$ correspond to the wavelength-dependent intensities of the transmitted light for TE- and TM-polarized light, respectively. Figure 8 compares the transmitted spectra and the obtained ISB absorption spectra for $\mathrm{GaN} /(\mathrm{Al}, \mathrm{Ga}) \mathrm{N}$ heterostructures grown on FS $a$-GaN and on $a$-GaN $/ r$-sap for different QW widths. A Lorentz fitting is used to fit the experimental ISB absorption curves and shows excellent fits for all cases. Clear shifts of the absorption peak to lower energies are observed with an increase in the QW width for both structures, resulting in a range of resonance wavelengths from about 250 to $300 \mathrm{meV}$ ( 4 to $5 \mu \mathrm{m}$ ). Table I summarizes experimental data displayed in Fig. 8 and compares it to theoretically computed values. Details of the calculations, which include many-body effects, are described in Ref. [12] and the Supplemental Material to this reference. The calculations are performed using a shooting method, in which a conduction-band offset of $551 \mathrm{meV}$ is used for $\mathrm{Al}_{0.4} \mathrm{Ga}_{0.6} \mathrm{~N} / \mathrm{GaN}$ system. The effective electron masses used in $\mathrm{Al}_{0.4} \mathrm{Ga}_{0.6} \mathrm{~N}$ and $\mathrm{GaN}$ are $0.264 m_{0}$ and $0.22 m_{0}$, respectively.

As expected from quantum-mechanical calculations, wider QWs result in ISB transitions with lower energies for structures grown on both substrates (FS $a$-GaN and $a$-GaN $/ r$-sap). However, for a given QW width, the co-loaded ISB structures grown on $a$-GaN $/ r$-sap show lower ISB transition energies than those grown on freestanding $a-\mathrm{GaN}$, which may be attributed to slightly thicker QWs (or different distributions of the Al content in the QBs) for the structures grown on $a-\mathrm{GaN} / r$-sap, as indicated in the STEM results of Fig. 5. The slight difference in growth rate could be due to the difference in the surface and bulk properties of the highly defective substrates compared with high-quality freestanding GaN. The ISB structures grown on $a$-GaN $/ r$-sap also show $10 \%-20 \%$ larger absorption linewidth compared with those grown on FS $a-\mathrm{GaN}$. However, despite the nearly 5 orders of magnitude difference in defect densities for the two sample sets, the ISB absorption linewidth is not significantly different between the samples grown on the two different substrates. Therefore, data demonstrate that, unlike band-to-band transition devices, the ISB absorption is very tolerant to extended defects. We note that these findings are consistent with recent results obtained for intersubband devices with $\mathrm{As}$ and $\mathrm{Sb}$ heterostructures grown on foreign substrates $[47,48]$. The larger ISB absorption linewidths for the structures grown on $a-\mathrm{GaN} / r$-sap can be partially explained by the surface morphology (Fig. 6) and interface-quality differences (Fig. 5). A systematic optimization of the growth conditions can improve the surface morphology of the $a$-GaN $/ r$-sap templates to further improve the interface quality and the ISB absorption properties. Given the manufacturing challenges of high-quality nonpolar GaN substrates, the low sensitivity of the ISB properties to extended defects may provide a cost-effective and scalable approach for producing efficient ISB emitters in the mid- to far-IR using a heteroepitaxial nonpolar III-nitride system on sapphire and Si.

\section{SUMMARY AND CONCLUSIONS}

We demonstrate a strong defect tolerance of $\mathrm{GaN} /$ $(\mathrm{Al}, \mathrm{Ga}) \mathrm{N}$ ISB structures grown on a nonpolar orientation. A comparison of two sets of samples grown on freestanding $a$-GaN and on $a$-GaN $/ r$-sapphire templates with at least 5 orders of magnitude difference, in terms of densities of extended defects (BSFs, TDs, PDs, PSFs), indicate only $5 \%$ difference in the peak ISB absorption energies and only $10 \%-20 \%$ difference in the ISB absorption linewidths. Structural data characterizations reveal the significant difference in material quality, as well as surface, interface abruptness, alloy disorder, and clustering for the two sample sets. Given the intrinsic properties of nonpolar group-III nitrides for IR optoelectronics, our results are promising for the development of cost-effective, scalable, and highly efficient mid- to far-IR optoelectronics using heteroepitaxial nonpolar III nitrides.

\section{ACKNOWLEDGMENTS}

This work is supported by the National Science Foundation (NSF) through Grants No. ECCS 1809691 and No. ECCS 1810318. Additional support is provided by the Air Force Office of Scientific Research (Program No. FA9550-19-1-10090), the DARPA NASCENT program, the Simons Foundation (Grant No. 601952, J.S.S.), and the NSF-RAISE program (Grant No. A007231601). The authors acknowledge support from the Solid-State Lighting and Energy Electronics Center (SSLEEC) at the University of California, Santa Barbara (UCSB). Samples are measured in the Microelectronics Research Center at the 
University of Texas at Austin, which is a member of the NSF National Nanotechnology Coordinated Infrastructure.

[1] J. Faist, F. Capasso, D. L. Sivco, C. Sirtori, A. L. Hutchinson, and A. Y. Cho, Quantum cascade laser, Science 264, 553 (1994).

[2] R. Paiella, Intersubband Transitions in Quantum Structures (McGraw-Hill Education, Boston, MA, 2006).

[3] H. C. Liu and F. Capasso, Intersubband Transitions in Quantum Wells: Physics and Device Applications (Academic Press, San Diego, CA, 1999).

[4] G. P. Agrawal and N. K. Dutta, Semiconductor Lasers (Van Nostrand Reinhold, New York, 1993), 2nd ed.

[5] J. Faist, Quantum Cascade Lasers (Oxford University Press, Oxford, 2013), 1st ed.

[6] B. S. Williams, Terahertz quantum-cascade lasers, Nat. Photonics 1, 517 (2007).

[7] H. Morkoç, Handbook of Nitride Semiconductors and Devices (John Wiley \& Sons, New York, 2009).

[8] E. Bellotti, K. Driscoll, T. D. Moustakas, and R. Paiella, Monte carlo simulation of terahertz quantum cascade laser structures based on wide-bandgap semiconductors, J. Appl. Phys. 105, 113103 (2009).

[9] G. Sun, J. B. Khurgin, and D. P. Tsai, Spoof plasmon waveguide enabled ultrathin room temperature $\mathrm{THz} \mathrm{GaN}$ quantum cascade laser: A feasibility study, Opt. Express 21, 28054 (2013).

[10] N. Suzuki and N. Iizuka, Feasibility study on ultrafast nonlinear optical properties of $1.55-\mu \mathrm{m}$ intersubband transition in AlGaN/GaN quantum wells, Jpn. J. Appl. Phys. 36, L1006 (1997).

[11] M. Beeler, E. Trichas, and E. Monroy, III-nitride semiconductors for intersubband optoelectronics: A review, Semicond. Sci. Technol. 28, 074022 (2013).

[12] M. Monavarian, J. Xu, M. N. Fireman, N. Nookala, F. Wu, B. Bonef, K. S. Qwah, E. C. Young, M. A. Belkin, and J. S. Speck, Structural and optical properties of nonpolar M- and a-plane $\mathrm{GaN} / \mathrm{AlGaN}$ heterostructures for narrow-linewidth mid-infrared intersubband transitions, Appl. Phys. Lett. 116, 201103 (2020).

[13] E. J. Roan and S. L. Chuang, Linear and nonlinear intersubband electroabsorptions in a modulation-doped quantum well, J. Appl. Phys. 69, 3249 (1991).

[14] K. Vijayraghavan, Y. Jiang, M. Jang, A. Jiang, K. Choutagunta, A. Vizbaras, F. Demmerle, G. Boehm, M. C. Amann, and M. A. Belkin, Broadly tunable terahertz generation in mid-infrared quantum cascade lasers, Nat. Commun. 4, 1 (2013).

[15] J. Lee, M. Tymchenko, C. Argyropoulos, P.-Y. Chen, F. Lu, F. Demmerle, G. Boehm, M.-C. Amann, A. Alù, and M. A. Belkin, Giant nonlinear response from plasmonic metasurfaces coupled to intersubband transitions, Nature 511, 7507 (2014).

[16] J. Lee, N. Nookala, J. S. Gomez-Diaz, M. Tymchenko, F. Demmerle, G. Boehm, M.-C. Amann, A. Alù, and M. A. Belkin, Ultrathin second-harmonic metasurfaces with record-high nonlinear optical response, Adv. Opt. Mater. 4, 664 (2016).
[17] N. Nookala, J. Lee, M. Tymchenko, J. S. Gomez-Diaz, F. Demmerle, G. Boehm, K. Lai, G. Shvets, M.-C. Amann, A. Alu, and M. Belkin, Ultrathin gradient nonlinear metasurface with a giant nonlinear response, Optica 3, 283 (2016).

[18] M. M. Fejer, S. J. B. Yoo, R. L. Byer, A. Harwit, and J. S. Harris Jr, Observation of Extremely Large Quadratic Susceptibility at 9.6-10.8 $\mu \mathrm{m}$ in Electric-Field-Biased AlGaAs Quantum Wells, Phys. Rev. Lett. 62, 1041 (1989).

[19] I. Vurgaftman, J. R. Meyer, and L. R. Ram-Mohan, Optimized second-harmonic generation in asymmetric double quantum wells, IEEE J. Quantum Electron. 32, 1334 (1996).

[20] C. Sirtori, F. Capasso, J. Faist, L. N. Pfeiffer, and K. W. West, Far-infrared generation by doubly resonant difference frequency mixing in a coupled quantum well twodimensional electron gas system, Appl. Phys. Lett. 65, 445 (1994).

[21] B. Corcoran, C. Monat, C. Grillet, D. J. Moss, B. J. Eggleton, T. P. White, L. O'Faolain, and T. F. Krauss, Green light emission in silicon through slow-light enhanced thirdharmonic generation in photonic-crystal waveguides, Nat. Photonics 3, 4 (2009).

[22] C. Wang, Z. Li, M.-H. Kim, X. Xiong, X.-F. Ren, G.-C. Guo, N. Yu, and M. Lončar, Metasurface-assisted phasematching-free second harmonic generation in lithium niobate waveguides, Nat. Commun. 8, 1 (2017).

[23] M. Celebrano, X. Wu, M. Baselli, S. Großmann, P. Biagioni, A. Locatelli, C. De Angelis, G. Cerullo, R. Osellame, B. Hecht, L. Duò, F. Ciccacci, and M. Finazzi, Mode matching in multiresonant plasmonic nanoantennas for enhanced second harmonic generation, Nat. Nanotechnol. 10, 5 (2015).

[24] N. Yu and F. Capasso, Flat optics with designer metasurfaces, Nat. Mater. 13, 2 (2014).

[25] A. V. Kildishev, A. Boltasseva, and V. M. Shalaev, Planar photonics with metasurfaces, Science 339, 1232009 (2013).

[26] R. W. Boyd, Nonlinear Optics (Academic Press, New York, 2008).

[27] G. Li, S. Chen, N. Pholchai, B. Reineke, P. W. H. Wong, E. Y. B. Pun, K. W. Cheah, T. Zentgraf, and S. Zhang, Continuous control of the nonlinearity phase for harmonic generations, Nat. Mater. 14, 6 (2015).

[28] M. Monavarian, A. Rashidi, and D. Feezell, A decade of nonpolar and semipolar III-nitrides: A review of successes and challenges, Phys. Status Solidi A 216, 1800628 (2019).

[29] D. F. Feezell, J. S. Speck, S. P. DenBaars, and S. Nakamura, Semipolar InGaN/GaN light-emitting diodes for high-efficiency solid-state lighting, J. Disp. Technol. 9, 190 (2013).

[30] A. E. Romanov, T. J. Baker, S. Nakamura, and J. S. Speck, Strain-Induced polarization in wurtzite III-nitride semipolar layers, J. Appl. Phys. 100, 023522 (2006).

[31] D. Feezell, Y. Sharma, and S. Krishna, Optical properties of nonpolar III-nitrides for intersubband photodetectors, J. Appl. Phys. 113, 133103 (2013).

[32] N. Suzuki and N. Iizuka, Effect of polarization field on intersubband transition in $\mathrm{AlGaN} / \mathrm{GaN}$ quantum wells, Jpn. J. Appl. Phys. 38, L363 (1999). 
[33] A. Pesach, E. Gross, C.-Y. Huang, Y.-D. Lin, A. Vardi, S. E. Schacham, S. Nakamura, and G. Bahir, Non-polar m-plane intersubband based InGaN/(Al)GaN quantum well infrared photodetectors, Appl. Phys. Lett. 103, 022110 (2013).

[34] C. Edmunds, J. Shao, M. Shirazi-HD, M. J. Manfra, and O. Malis, Terahertz intersubband absorption in non-polar mplane AlGaN/GaN quantum wells, Appl. Phys. Lett. 105, 021109 (2014).

[35] C. B. Lim, A. Ajay, C. Bougerol, B. Haas, J. Schörmann, M. Beeler, J. Lähnemann, M. Eickhoff, and E. Monroy, Nonpolarm-Plane GaN/AlGaN heterostructures with intersubband transitions in the 5-10 THz band, Nanotechnology 26, 435201 (2015).

[36] T. D. Moustakas and R. Paiella, Optoelectronic device physics and technology of nitride semiconductors from the UV to the terahertz, Rep. Prog. Phys. 80, 106501 (2017).

[37] T. Kotani, M. Arita, and Y. Arakawa, Observation of mid-infrared intersubband absorption in non-polar m-plane AlGaN/GaN multiple quantum wells, Appl. Phys. Lett. 105, 261108 (2014).

[38] J. Xu, M. Monavarian, N. Nookala, M. N. Fireman, K. S. Qwah, J. S. Speck, and M. A. Belkin, in 2020 Conference on Lasers and Electro-Optics (CLEO) (2020), pp. 1-2.

[39] M. Funato, M. Ueda, Y. Kawakami, Y. Narukawa, T. Kosugi, M. Takahashi, and T. Mukai, Blue, Green, and amber InGaN/GaN light-emitting diodes on semipolar \{11-22\} GaN bulk substrates, Jpn. J. Appl. Phys. 45, L659 (2006).

[40] K. Fujito, S. Kubo, and I. Fujimura, Development of bulk GaN crystals and nonpolar/semipolar substrates by HVPE, MRS Bull. 34, 313 (2009).

[41] See the Supplemental Material at http://link.aps.org/supple mental/10.1103/PhysRevApplied.16.054040 for descrip- tions of postprocessing of experimental data and a more detailed discussion of design considerations, including the effect of doping and number of QWs on the ISB properties.

[42] L. Rigutti, B. Bonef, J. Speck, F. Tang, and R. A. Oliver, Atom probe tomography of nitride semiconductors, Scr. Mater. 148, 75 (2018).

[43] M. N. Fireman, B. Bonef, E. C. Young, N. Nookala, M. A. Belkin, and J. S. Speck, Strain compensated superlattices on M-plane gallium nitride by ammonia molecular beam epitaxy, J. Appl. Phys. 122, 075105 (2017).

[44] K. Thompson, D. Lawrence, D. J. Larson, J. D. Olson, T. F. Kelly, and B. Gorman, In situ site-specific specimen preparation for atom probe tomography, Ultramicroscopy $\mathbf{1 0 7}$, 131 (2007).

[45] M. D. Craven, S. H. Lim, F. Wu, J. S. Speck, and S. P. DenBaars, Structural characterization of nonpolar (1120) a-plane GaN thin films grown on $\left(11^{-} 02\right)$ r-plane sapphire, Appl. Phys. Lett. 81, 469 (2002).

[46] E. E. Fullerton, I. K. Schuller, H. Vanderstraeten, and Y. Bruynseraede, Structural refinement of superlattices from X-ray diffraction, Phys. Rev. B 45, 9292 (1992).

[47] H. Nguyen-Van, A. N. Baranov, Z. Loghmari, L. Cerutti, J.-B. Rodriguez, J. Tournet, G. Narcy, G. Boissier, G. Patriarche, M. Bahriz, E. Tournié, and R. Teissier, Quantum cascade lasers grown on silicon, Sci. Rep. 8, 1 (2018).

[48] R. Go, H. Krysiak, M. Fetters, P. Figueiredo, M. Suttinger, J. Leshin, X. M. Fang, J. M. Fastenau, D. Lubyshev, A. W. K. Liu, A. Eisenbach, M. J. Furlong, and A. Lyakh, Room temperature operation of quantum cascade lasers monolithically integrated onto a lattice-mismatched substrate, Appl. Phys. Lett. 112, 031103 (2018). 